\title{
HIGH SPATIAL AND \\ TEMPORAL RESOLUTION VISIBLE SPECTROSCOPY OF THE PLASMA EDGE IN DIII-D
}

\author{
by \\ P. Gohil, K.H. Burrell, \\ R.J. Groebner, and R.P. Seraydarian
}

This is a preprint of a paper to be presented at the 8th Topical Conference on High Temperature Plasma Diagnostics, May 7-10, 1990, Hyannis, Massachusetts, and to be printed in the Proceedings.

\author{
Work supported by \\ Department of Energy \\ Contract DE-AC03-89ER51114
}
GENERAL ATOMICS PROJECT 3466 OCTOBER 1990

\section{GENERAL ATOMICS}




\section{High spatial and temporal resolution visible spectroscopy of the plasma edge in DIII-D}

P. Gohil, K.H. Burrell, R.J. Groebner, AND R.P. SERAYDARIAN

General Atomics, San Diego, California 92186-9084

In DIII-D, visible spectroscopic measurements of the He II $468.6 \mathrm{~nm}$ and C VI $529.2 \mathrm{~nm}$ Doppler broadened spectral lines, resulting from charge exchange recombination interactions between beam neutral atoms and plasma ions, are performed to determine ion temperatures, and toroidal and poloidal rotation velocities. The diagnostics system comprises 32 viewing chords spanning a typical minor radius of $63 \mathrm{~cm}$ across the midplane, of which 16 spatial chords span $11 \mathrm{~cm}$ of the plasma edge just within the separatrix. A temporal resolution of $260 \mu$ s per time slice can be obtained as a result of using MCP phosphors with short decay times and fast camera readout electronics. Results from this system will be used in radial electric field comparisons with theory at the L-H transition and ion transport analysis. 


\section{INTRODUCTION}

High spatially and temporally resolved measurements of ion temperature and poloidal and toroidal rotation velocities at the plasma edge provide details on the edge plasma radial electric field, $E_{r}$, especially with regard to the phenomenology of the L-H transition. ${ }^{1,2}$ Furthermore, detailed measurements are required for ion thermal transport analysis. ${ }^{3,4}$ These requirements have been the driving force behind the upgrade to the charge exchange recombination (CER) diagnostic system for DIII-D. The previous system consisted of 16 spatial chords viewing across the $63 \mathrm{~cm}$ plasma minor radius with a spatial resolution of 3-4 cm per spatial point. ${ }^{5}$ The temporal resolution was $5 \mathrm{msec}$ with measurements made in both the toroidal and poloidal direction. Multichannel detection of visible plasma emission provided spectrally resolved data for determination of Doppler broadened and Doppler shifted spectral lines resulting from charge exchange recombination interactions.

The major improvements to the CER diagnostic system have beer: the addition of high spatially resolved viewing chords at the plasma edge, in both the poloidal and toroidal directions; increased temporal resolution capability to $260 \mu \mathrm{sec}$ per timeslice, and; redistribution of all the viewing chords for optimum transport analysis.

\section{SYSTEM HARDWARE}

The description of the hardware can be separated into two sections: (a) the hardware components associated with the vacuum vessel and the related chordal layout within the plasma configurations; (b) the spectral dispersion and detection of the CER emission from the plasma. 
Beginning with Section (a), Fig. 1(a) shows a plan view of the chordal geometry in the plasma through several neutral beams. There are a total of 32 chords, 15 in the vertical and 17 in the horizontal plane. Thirteen of these chords are designated as central chords and observe from about $10 \mathrm{~cm}$ to $50 \mathrm{~cm}$ from the plasma center. There are also sixteen chords which observe across $11 \mathrm{~cm}$ of the plasma edge across the separatrix at the midplane. These edge chords are arranged in sets of poloidally and toroidally viewing directions of eight chords each, but interdispersed so adjacent spatial points alternate between the two sets [Fig. 1(b)]. This arrangement provides for high resolution for $T_{i}(r)$ and $d T_{i}(r) / d r$ measurements, but also gives good spatial resolution for edge poloidal and toroidal rotation velocities within $10 \mathrm{~cm}$ of the separatrix. The edge poloidal chords are viewed by $1500 \mu \mathrm{m}$ silica core optical fibers whereas the toroidal chords use $750 \mu \mathrm{m}$ core fibers bundled in pairs and aligned vertically when viewing through the plasma so as to minimize the horizontal extent of the observed region. The plasma emission is directed onto the fibers by flat aluminum-coated mirrors and focussed using single convex lenses. The optics are coated for enhanced efficiency in the spectral region of interest. The magnification of the optics and separation of the fibers in the image plane was carefully determined to provide clear separation of the poloidal and toroidal chords along the $11 \mathrm{~cm}$ at the plasma edge at the midplane. There are also two sets of chords which view essentially the same major radii in both the poloidal and toroidal direction. These secondary chords are used as references against chords at similar radii, but viewing in the opposite direction, so poloidal and toroidal rotation velocity measurements in order to establish a zero of rotation. 




Fig. 1(a). Plan view of DIII-D indicating the trajectories of the toroidally viewing chords through the neutral beams. The dots indicate the vertical views through the beams. The edge views are shown in more detail in Fig. 1(b).



Fig. 1(b). Edge poloidal and toroidal viewing arrays along the neutral beam centerline. The circles represent the images of the optical fibers through the viewing optics. The inside points of the arrays contain two sets of views - one being used as a reference against views in the opposite direction as a check for absolute rotation velocity measurements. 
The spatial localization of the plasma emission is determined from the convolution of the neutral beam cross section, the curvature of the toroidal and poloidal flux surfaces and the geometry and cross-section of the viewing chords. The viewing chords are then arranged for the highest spatial resolution possible for a given position along the major radius given the constraint of accessibility within the vacuum vessel. Flux plots were generated for various single-null and double-null plasma configurations and the geometry of the viewing chords was defined so as to produce the minimum uncertainty along the flux surface through the neutral beam of observation. This condition is satisfied best when the viewing chord is at a tangency to the flux surface of irterest. The full width half maximum (FWHM) of the neutral beam cross-sectional dimensions are $14 \mathrm{~cm}$ and $30 \mathrm{~cm}$ in the horizontal and vertical directions, respectively. Furthermore, the radius of curvature of the flux surfaces along the toroidal direction is larger than the radius of curvature in the poloidal direction. Therefore the spatiai resolution for the poloidal chords is less than for the toroidal chords with the central chords being worse than the edge chords as a result of the lowering radius of curvature of the flux surfaces. Typically the central poloidal chords have spatial resolution of $\pm 1.5 \mathrm{~cm}$, whereas the edge poloidal chords have a resolution of $\pm 1 \mathrm{~cm}$. The central toroidal chords have a resolution of about $\pm 1 \mathrm{~cm}$, whereas the edge toroidal chords have the besi spatial resolution of $\pm 0.5 \mathrm{~cm}$ by virtue of the combination of the lowest neutral beam vraist, the smaller core size of optical fibers used and smaller magnetic flux c!rvature. 
The plasma emission is directed through up to 19 meters of optical fibers from the various viewing optics to an area outside the neutron shield wall. The light is observed through three 0.75 meter f/6.8 spectrometers and one 1 meter Czerny-Turner spectrometer with the 1 meter instrument being one of the central spectrometers; each spectrometer observes eight spatial chords. Each spectrometer contains a $1200 \mathrm{~g}$ $\mathrm{mm}^{-1}$ grating with the gratings, which observe the central chords, blazed at around the wavelengths for the first order of the spectral line of helium or carbon and the gratings observing the edge chords blazed for the second order of these lines. The exit plane of each spectrometer then contains eight spectrally dispersed bands representing the spatial channels arranged one above the other with a $3 \mathrm{~mm}$ center-to-center spacing. The spectral light of the edge chords is amplified through a proximity focussed dual plate intensifier tube coated with P46 phosphor on the output side, which has a fast decay time of the order of microseconds. The image from each spectrometer is then directed by a high density coherent optical fiber coupling onto two 1024 pixel reticon arrays with four spatial channels arranged along the length of each array. With a pixel spacing of $25 \mu \mathrm{m}$ and 256 pixels per spectrum for each spatial chord the overall spectral resolution of the detection system is $0.28 \AA /$ pixel and $0.21 \AA /$ pixel for the central chords and $0.11 \AA /$ pixel each for the edge chords. The reticon readout time is $500 \mathrm{~ns} /$ pixel for the edge chords, which by using a two phase clock and simultaneous readout of even and odd pixels gives a total readout time of $260 \mu$ s per reticon including the dead time between readouts. ${ }^{6}$ The use of fast readout electronic circuitry and MCP phosptor effectively allow for a minimum integration time of $260 \mu \mathrm{sec}$. However, the actual integration time is limited by the brightness of the spectral line. The 
acquired data is transferred via a microvax to the DIII-D Modcomp data acquisition computer.

\section{CER OBSERVATIONS}

The spectroscopic measurements of the Doppler broadened and shifted spectral lines provides knowledge of $T_{i}$ and $v_{\theta}$ and $v_{\perp}$ profiles provided the emission is localized at given points of observation. The signal enhancement results from CER interactions between the energetic deuterium neutrals from the heating beam and fully stripped helium or carbon ions. For the resulting hydrogen.like ionic species, the observed radiation is $468.6 \mathrm{~nm}(n=4 \rightarrow 3)$ for He II and $529.2 \mathrm{~nm}(n=8 \rightarrow 7)$ for C VI. Sufficient helium is left over from helium glow discharges in DIII-D to provide adequate ionic concentrations. Similarily for carbon, the large area of carbon tile covering within the vacuum vessel provides enough carbon for a good signal at moderate integration times of 5 msec.

Carbon CER emission is detected for the edge measurements where the species concentration is high, but helium CER emission is used for the central chords since the carbon signal becomes weak in this region. However, the He II spectrum is complicated by a cold, intense line from the edge which is also fitted in the analysis. 


\section{DATA ANALYSIS}

The determination of $T_{i}, v_{\phi}$, and $v_{\perp}$ is performed by a nonlinear least squares fit of the spectral data according to a Gaussian distribution convolved with the instrumental response function. This is performed for every spectral peak in the observed spectrum including peaks resulting from the presence of impurity lines. The fit parameters returned after a successful convergence are carefully checked particularly with regard to the departure of the theoretical fit from the actual data points along the array of pixels. The instrumental response is obtained from analysis of the Hg I $546.1 \mathrm{~nm}$ line from a low pressure mercury line such that any pressure broadening is below the intrinsic instrumental linewidth, which is found to be well fitted by the sum of three Gaussian line shapes. Figure 2 shows the ion temperature $T_{i}$ profile as a function of the minor radius. The region in the edge is shown enlarged to more clearly indicate the spatial resolution there. Figure 3 shows the $v_{\phi}$ and $v_{\perp}$ as a function of major radius.

\section{ACKNOWLEDGEMENT}

This work was supported by the U.S. Department of Energy under Contract No. DE-AC03-89ER511114. 

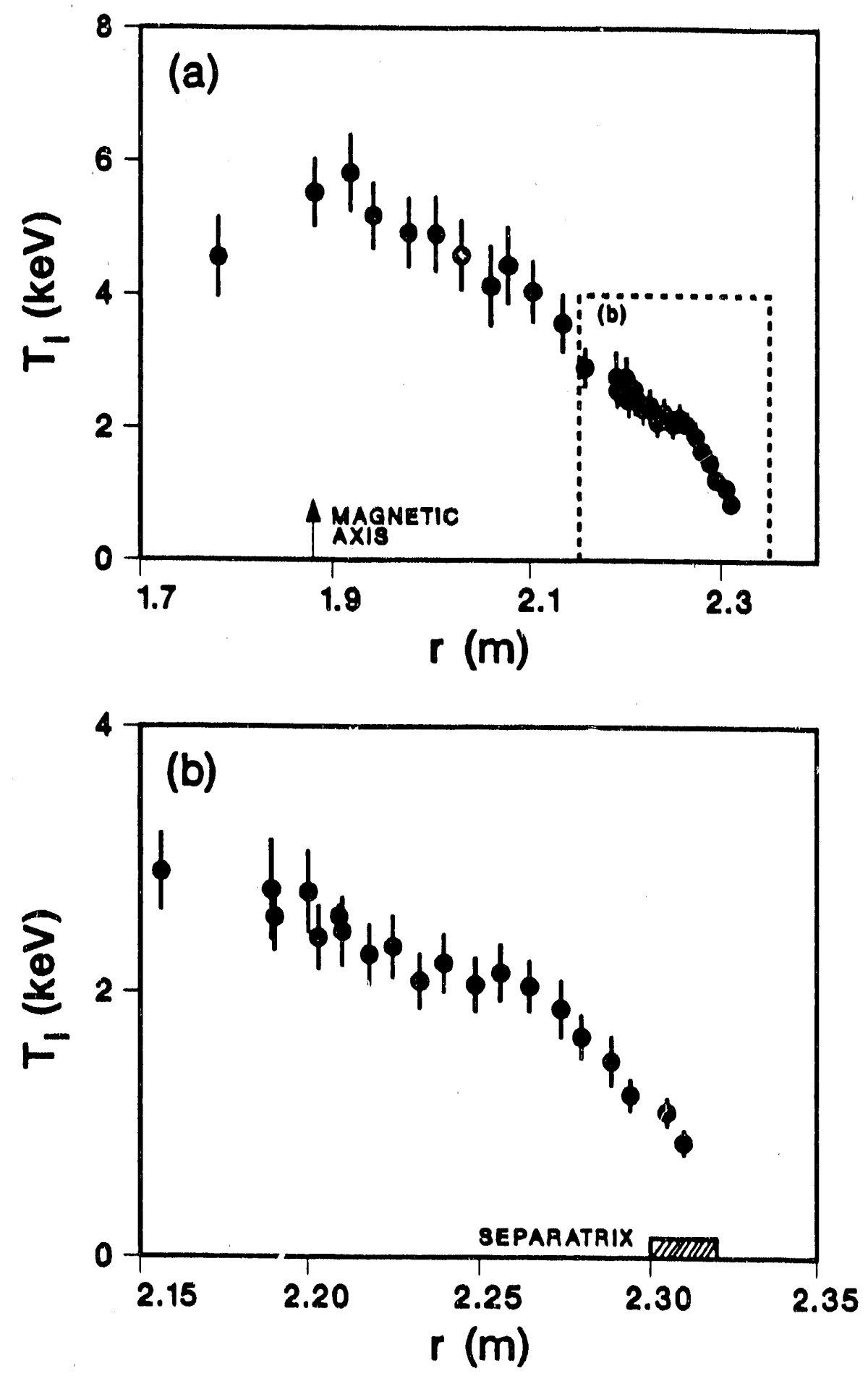

Fig. 2. Ion temperature profile as a function of the major radius of the plasma. 


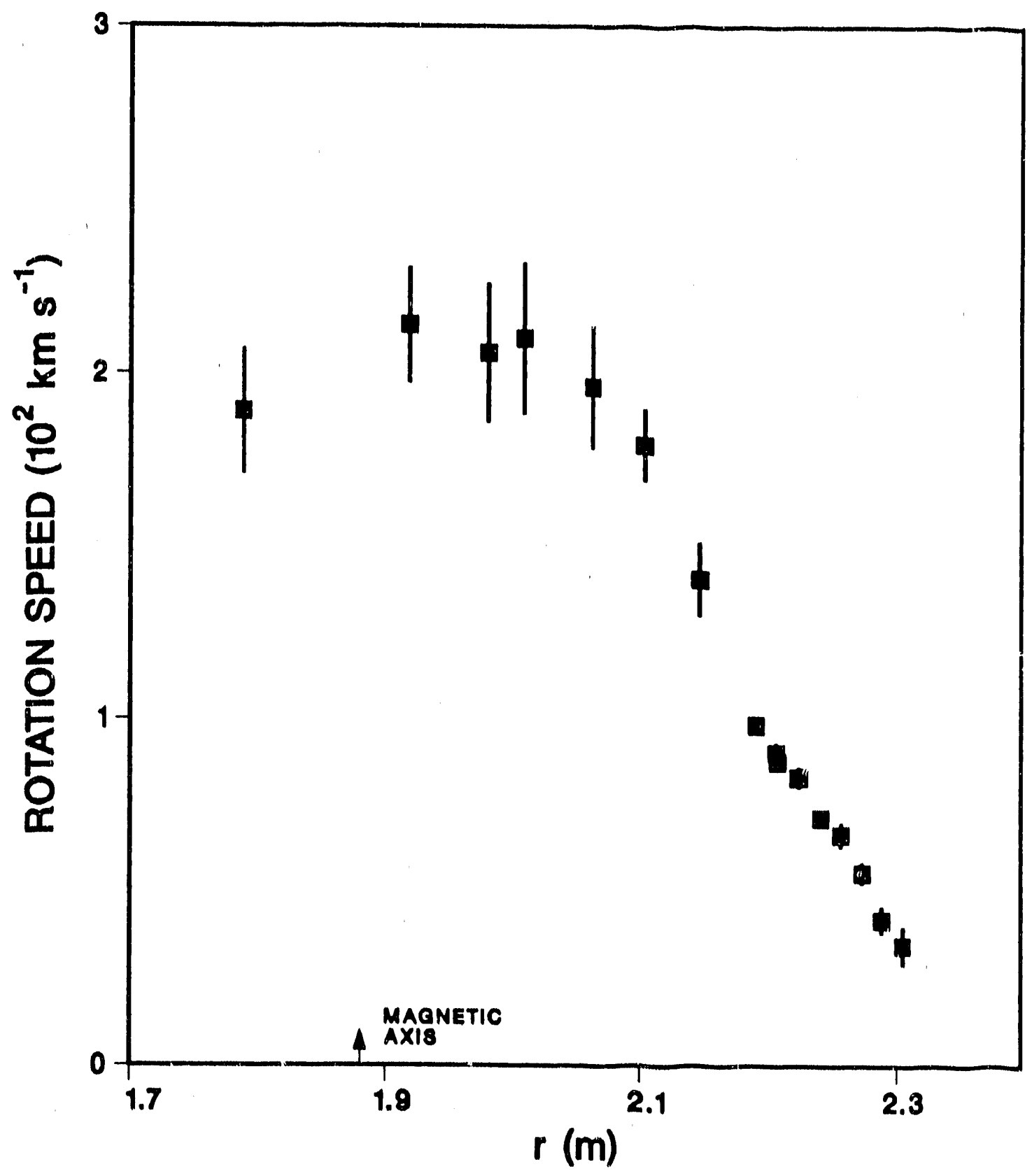

Fig. 3. The toroidal rotation velocity, $v_{\phi}$ as a function of the major radius of the plasma. 


\section{REFERENCES}

1 R. J. Groebner, K. H. Burrell, and R. P. Seraydarian, Phys. Rev. Lett. 64, 3015 (1990).

2 K. C. Shaing, and E. C. Crume, Jr., Phys. Rev. Lett. 63, 2369 (1989).

3 R. J. Groebner, W. Pfeiffer, F. P. Blau, K. H. Burrell, E. S. Fairbanks, R. P. Seraydarian, H. St. John, R. E. Stockdale, Nucl. Fusion 26, 543, (1986).

4 R. J. Fonck, et al., Phys. Rev. Lett. 63, 520, (1989).

5 R. P. Seraydarian, K. H. Burrell, and R. J. Groebner, Rev. Sci. Instru. 59, 1531, (1988).

${ }^{\theta}$ J. S. Haskovec, G. Bramson, N. H. Brooks, and M. Perry, General Atomics report GA-A19753, December 1989. 


